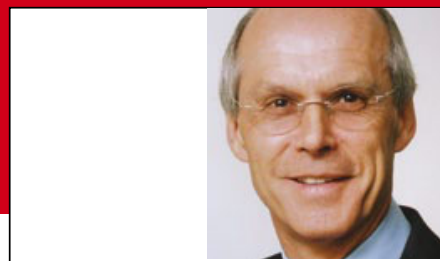

Prof. Dr. med. H. S. FüeßI Isar-AmperKlinikum, KI. MünchenOst, Haar

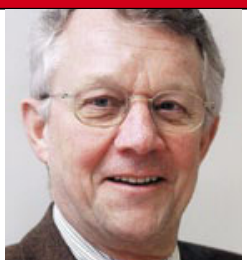

Prof. Dr. med. H. Holzgreve Internist, Kardiologische Praxis, München

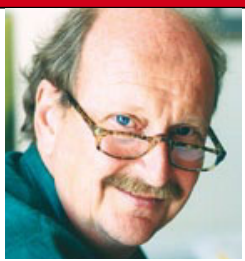

Prof. Dr. med. E. Ernst

Peninsular Medical School, University of Exeter/UK

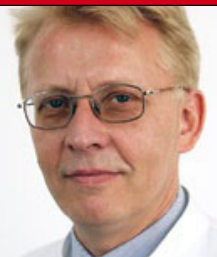

Prof. Dr. med. K. Rasche

Helios Klinikum

Barmen,

Wuppertalz

\title{
Ist Komplementärmedizin kosteneffektiv?
}

\begin{abstract}
Kann die Komplementärmedizin dazu beitragen, die Kosten im Gesundheitswesen zu senken? Eine umfangreiche australische Studie kommt hier zu einem überwiegend positiven Ergebnis.
\end{abstract}

— Im Jahr 2009 beauftragte das Australische National Institute of Complementary Medicine eine professionelle Organisation namens Access Economics, eine Kosten-Evaluation der Komplementärmedizin zu erstellen. Das Ergebnis liegt nun als Online-Publikation vor. Die Autoren haben sich auf fünf eng gefasste Teilbereiche beschränkt:

(1) Akupunktur gegen Rückenschmerzen,

2 Johanniskraut bei leichten und mittelschweren Depressionen,

(3) Omega-3-Fettsäuren zur Prävention einer KHK,

(4) Omega-3-Fettsäuren zur Reduktion von NSAR bei rheumatoider Arthritis, und

5 Phytodolor bei Arthrose.

Die umfangreichen Analysen (ca. 150 Seiten) zeigen, dass Kosteneffektivität für alle Teilbereiche, außer Omega-3 bei rheumatoider Arthritis, gegeben ist.

Die Akupunktur bei Kreuzschmerzen war kosteneffektiv, wenn sie adjuvant zu-

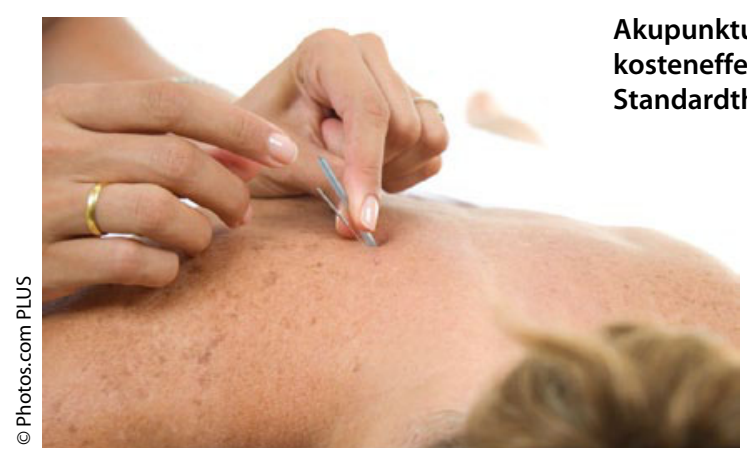

Akupunktur bei Rückenschmerzen: kosteneffektiv, aber kein Ersatz für die tandardtherapie.

zu beweisen, dass durch solche Verfahren die Gesundheitskosten zu senken sind, dann wäre die Diskussion über Sinn oder Unsinn dieser Methoden mit einem Schlag beendet. Die hier vorgelegten Analysen sind gut gemacht und kommen zu einem

sätzlich zur Standardtherapie genutzt wurde, sie war nicht kosteneffektiv, wenn sie statt einer Standardtherapie eingesetzt wurde. Beim Johanniskraut ergab sich die Kosteneffektivität aus dem Dosispreis (im Vergleich zu Standardantidepressiva). Omega-3-Fettsäuren reduzieren bei Patienten mit bekannter KHK die Morbidität und Mortalität. Als adjuvante Therapie sind sie hier hochgradig kosteneffektiv. Bei rheumatoider Arthritis tragen sie hingegen nicht zur Reduzierung von nicht steroidalen Antirheumatika bei. Phytodolor bei Arthrose ist preisgünstiger als Diclofenac.

\section{Kommentar}

Die Kosteneffektivität der Komplementärmedizin (KM) ist seit vielen Jahren ein heißes Eisen. Gelänge es den Anhängern der KM für die KM positiven Urteil. Fast alle systematischen Reviews zum Thema ziehen dagegen negative Schlüsse. Wie ist diese Diskrepanz zu erklären? Ganz einfach, der Schlüssel liegt hier in der Selektion der Teilbereiche. Diese beschränken sich im vorliegenden Fall mit viel Bedacht auf Methoden und Indikationen, die besser belegt sind als alle anderen KM-Verfahren. Manch kritischer Leser mag sogar fragen, ob Omega-3-Fettsäuren überhaupt zur KM zu rechnen sind. Mein Fazit lautet daher, dass die Kosteneffektivität der $K M$ in einzelnen, kleinen Teilbereichen gut belegt ist-aber nur in diesen.

E. ERNST .

- National Institute of Complementary Medicine Cost effectiveness of complementary Medicine. August 2010. http://www.nicm.edu.au/images/ stories/research/docs/cost_effectiveness_cm_ ae_2010.pdf 